\title{
Free open access to liberate and unleash neglected science
}

King-Hwa Ling ${ }^{1,2, *}$, Noraishah Mydin Abdul Aziz ${ }^{3}$ and Norshariza Nordin ${ }^{1,2}$

${ }^{1}$ Department of Biomedical Science, Faculty of Medicine and Health Sciences, Universiti Putra Malaysia, Selangor, Malaysia.

${ }^{2}$ Genetics and Regenerative Medicine Research Centre, Faculty of Medicine and Health Sciences, Universiti Putra Malaysia, Selangor, Malaysia.

${ }^{3}$ Department of Parasitology, Faculty of Medicine, University of Malaya, Kuala Lumpur, Malaysia.

* Correspondence: Ikh@upm.edu.my; Tel.: +603-89472564

Published Online: 4 March 2018

DOI: https://doi.org/10.31117/neuroscirn.v1i1.4

Keywords: journal publishing; open access; neuroscience; neglected science

C2018 by Ling et al for use and distribution in accord with the Creative Commons Attribution (CC BY-NC 4.0) license (https://creativecommons.org/licenses/by-nc/4.0/), which permits unrestricted non-commercial use, distribution, and reproduction in any medium, provided the original author and source are credited.

Open access has become a choice for scientific publication. In many countries, open access publication has been made compulsory for publicly funded studies. Since 2013, government-funded research paper in United States must be made freely available within 12 months of publication [1] ]. Such policy will ensure maximum accessibility and circulation of published articles. While the scientific community is benefited at large due to free access and distribution of published materials, open access publication is never free due to the various costs involved in the production, permanent archival and digitalisation of scholarly articles. Someone must foot the costs for web hosting, online marketing, database maintenance, copyediting, proofreading, indexing and permanent archiving of every single accepted article. In many instances, the submitting author will have to pay article processing charges, which range from USD500 to USD5000 depending on the reputation of the journal [2]. Source of funding is largely research grants-derived that do not last forever. With limited funds, authors are forced to prioritise their publications depending on the availability of funding to pay for article processing charges.

With the harsh "publish or perish" reality in the scientific community, numerous research remain unpublished due to the current trend where only positive and comprehensive studies are preferably considered by various established journals. Many single experiment observations are important but do not find a place in the highly cited high impact journals. In addition, most institutions do not provide sufficient financial support for publication charges leading to a deadlock situation where many small studies remain trapped within the laboratory notebooks.

Neuroscience Research Notes or NeurosciRN aims to serve as the platform to accommodate these findings while maintaining the fundamental purposes of hypothesis formation and testing in science particularly in neuroscience. NeurosciRN is managed and run by a group of dedicated scientist volunteers. The journal will 
serve as a platform for neglected science; science that was deemed too small, incomplete or bear no positive findings. NeurosciRN will serve as a new home to neglected science as long as the study was properly performed, and the data or story is adequately interpreted or told. Nothing is too small for us. Only good science or bad science.

NeurosciRN will liberate and uncover neglected science that scientists have spent substantial time, effort and expenditure. Why keep them in the laboratory notebook when they could be showcased and made known to everyone? Previously, funding has been the main hurdle in preventing the publication of small findings and therefore we make it possible by enabling everyone to access, read and submit to NeurosciRN without any fees. As many would wonder and question the sustainability of this truly free open access journal, we will try our best level to minimise the publishing cost to suit its purpose. So much of volunteerism, we welcome any party or individual to any monetary contribution or any of sort in helping us to uphold this noble intention making NeurosciRN, truly a journal from scientists to scientists.

Above all else, it is amazing that we have managed to garner the support of so many distinguished scientists from around the world to support NeurosciRN. We, the founding editors of NeurosciRN hereby promise that this journal will uphold the sacred tenets of good science, that we will screen the manuscripts with the highest standards and we will not falter at making decisions which should not be influenced neither by fear nor favour.

\section{References}

1. Van Noorden R. US science to be open to all. Nature. 2013; 494(7442): 414-415. https://doi.org/10.1038/494414a

2. Van Noorden R. Open access: The true cost of science publishing. Nature. 2013; 495(7442): 426-429. https://doi.org/10.1038/495426a 